\title{
POINT PARTITION NUMBERS AND GIRTH
}

\author{
R. J. COOK
}

ABSTRACT. In recent papers D. R. Lick and A. T. White have introduced point partition numbers as generalizations of the chromatic number and the point-arboricity of a graph. In particular they proved that an analogue of Heawood's theorem holds for the point partition numbers. In the present paper it is shown that the bounds provided by their result may be improved for graphs of large girth. Finally, using a method of Erdös, it is shown that there exist graphs with large girth and large point-partition number.

1. Introduction. For a graph $G$, let $\delta(G)$ denote the minimum degree of the vertices of $G$. A graph $G$ is said to be $k$-degenerate if every induced subgraph $H$ of $G$ satisfies the inequality $\delta(H) \leq k$. The point partition number of order $k$ of the graph $G$, denoted by $\rho_{k}(G)$, is the minimum number of subsets into which the vertex set of $G$ can be partitioned so that each subset induces a $k$-degenerate subgraph of $G$. The point partition numbers were introduced by Lick and White [7], [8] as generalizations of the chromatic number and the point-arboricity of a graph. The parameters $\rho_{0}(G)$ and $\rho_{1}(G)$ are respectively the chromatic number and the point-arboricity of $G$. For definitions not given here, see [4].

For a graph $G$ of given positive genus, Heawood [5] and Kronk [6] have obtained bounds for the chromatic number and the point-arboricity of a graph $G$. These results were generalized to point partition numbers by Lick and White [8]. In [1], [2] the author has shown that improved bounds may be obtained for the chromatic number and the point-arboricity of a graph $G$ if the girth of $G$ is large. The object of this note is to show that these methods extend to point partition numbers. We shall henceforth suppose that $k$ is a fixed nonnegative integer and write $\rho$ for $\rho_{k}$.

Theorem 1. Let $G$ be a graph of positive genus $\gamma$, having no triangles. Then

$$
\rho(G) \leq 1+[2(1+\sqrt{ } \gamma) /(k+1)]
$$

where $[x]$ denotes the integer part of $x$.

Received by the editors November 28, 1972 and, in revised form, June 10, 1973 and February 4, 1974.

AMS (MOS) subject classifications (1970). Primary 05C 99, 05C10, $05 \mathrm{C} 15$.

Key words and phrases. Point partition numbers, girth, genus. 
Heawood's bounds on the chromatic number of nonspherical surfaces have proved to be sharp, however the bounds provided by Theorem 1 are not sharp.

Erdös [3] has shown that for every $n$ and $t$ there exists a graph $G=$ $G(n, t)$ of chromatic number $n$ and girth at least $t$.

Theorem 2. For every $n$ and $t$ there is a graph $G=G(n, t, k)$ with $\rho(G)=n$ and girth at least $t$.

However, for graphs of given genus it is possible to bound $\rho(G)$ in terms of the girth $g(G)$.

Theorem 3. Suppose that $k \geq 2$, let $G$ be a graph of positive genus $\gamma$ and let $t$ be a positive integer such that $g(G) \geq 1+4 t$. Let $n$ be a positive integer and put $h=(k+1) n$. If

$$
g(G)>2\left(2(\gamma-1)+h(h-1)^{t}\right) /\left((h-2)(b-1)^{t}\right)
$$

then $\rho(G) \leq n$.

For $k=0$ and $k=1$, analogous results were proved in [1], [2]. Clearly, for any fixed genus $\gamma$ and all large $k \geq k_{0}(\gamma)$ we have $\rho(G)=1$ for all graphs $G$ of genus $\gamma$. Also for any fixed $k$ and $\gamma$, for all graphs $G$ of sufficiently large girth we have $\rho(G)=1$. Taking $t=\left\{\log _{k} 4 y\right\}$, where $\{x\}$ denotes the least integer not less than $x$, we have the following corollary to Theorem 3 .

Theorem 4. Let $G$ be a graph of positive genus $\gamma$ and let $k \geq 2$. If $g(G)$ $\geq 1+4\left\{\log _{k} 4 \gamma\right\}$ then $\rho(G)=1$.

For planar graphs we combine the results of Lick and White [8], a theorem of Grötzsch, see [4, p. 131], and the methods of Theorem 1 to give the following bounds for $\rho(G)$.

\begin{tabular}{|c|c|c|c|c|c|c|}
\hline$g(G)$ & 0 & 1 & 2 & 3 & 4 & $\geq 5$ \\
\hline 3 & 5 & 3 & 3 & 2 & 2 & 1 \\
\hline$\geq 4$ & 3 & 2 & 2 & 1 & 1 & 1 \\
\hline
\end{tabular}

2. Proof of Theorem 1. We embed $G$ in an orientable surface of genus $\gamma(G)$ and suppose that $G$ has $p$ vertices, $q$ edges and $r$ faces. 
Lemma 1 (Euler's formula). Let $G$ be a graph of genus $\gamma$, then

$$
2-2 \gamma=p-q+r \text {. }
$$

Further, if $G$ contains no triangle then

$$
\gamma \geq 1-p / 2+q / 4
$$

See, for example, [4, pp. 117-118].

If $v$ is a vertex of $G$ then by $G-v$ we mean the graph obtained from $G$ by removing $v$ and all edges incident with $v$. Clearly $\rho(G-v) \leq \rho(G)$. A graph $G$ is called critical here if $\rho(G-v)<\rho(G)$ for all vertices $v$ of $G$. An $n$-critical graph is a critical graph with $\rho(G)=n$, every graph with $\rho(G)$ $=n$ contains an $n$-critical subgraph.

Lemma 2. If $G$ is an $n$-critical graph then $\delta(G) \geq(k+1)(n-1)$.

This is Theorem 4 of [7].

Let $\rho(G)=n$ and suppose first that $G$ is $n$-critical. From Lemmas 1 and 2 we have

$$
8 \gamma-8+4 p \geq 2 q \geq(k+1)(n-1) p .
$$

Lemma 3. If $G$ contains no triangles then $q \leq p^{2} / 4$.

This is due to Turán [9].

Combining Lemma 3 with the dexter inequality in (6) we see that $p \geq$ $2(k+1)(n-1)$. Then (6) gives $4 y-4 \geq((k+1)(n-1)-4)(k+1)(n-1)$ so that

$$
(k+1)^{2} n^{2}-2(k+1)(k+3) n+(k+1)^{2}+4(k+2-\gamma) \leq 0,
$$

which gives (1).

If $G$ is not $n$-critical then it possesses an $n$-critical subgraph $H$ which also has no triangles, and the bound (1) holds for $\rho(H)$.

3. Proof of Theorem 2. The following proof is based on the method of Erdös [3]. Let $t$ be given and let $\epsilon$ satisfy $0<\epsilon<t^{-1}$ For any large integer $s$ we put $m=\left[s^{1+\epsilon}\right]$ and $p=\left[s^{1-\eta}\right]$ where $0<\eta<1 / 2 \epsilon$. Let $K_{s}$ be the complete graph on $s$ vertices and let $K_{p}$ be any of its complete subgraphs having $p$ vertices. We can choose $K_{p}$ in $\left(\begin{array}{l}s \\ p\end{array}\right)$ different ways. We put

$$
M=\left(\begin{array}{l}
m \\
2
\end{array}\right), \quad S=\left(\begin{array}{l}
s \\
2
\end{array}\right), \quad P=\left(\begin{array}{l}
p \\
2
\end{array}\right) \text { and } C=\left(\begin{array}{l}
S \\
m
\end{array}\right) \text {. }
$$

Let

$$
G_{x}, \quad x=1,2, \ldots, C,
$$


be the subgraphs of $K_{s}$ having $m$ edges. By "almost all $G_{x}$ " we mean that the number of exceptions is $o(C)$. Erdös has shown that almost all $G_{x}$ contain fewer than $s / t$ closed circuits of length not exceeding $t$. We now consider the number of edges in $G_{x} \cap K_{p}$. Following Erdös, for any fixed $K_{p}$ we see that the number of graphs $G_{x}$ containing not more than $(k+1) s$ edges of $K_{p}$ equals

$$
\begin{aligned}
\sum_{i=0}^{(k+1) s}\left(\begin{array}{c}
P \\
i
\end{array}\right)\left(\begin{array}{c}
S-P \\
m-i
\end{array}\right) & <((k+1) s+1)\left(\begin{array}{c}
P \\
(k+1) s
\end{array}\right)\left(\begin{array}{c}
S-P \\
m
\end{array}\right) \\
& <p^{2(k+1) s}\left(\begin{array}{c}
S-M \\
m
\end{array}\right)<C p^{2(k+1) s} \exp \left(\frac{-m p^{2}}{s^{2}}\right)
\end{aligned}
$$

The number of possible choices for $K_{p}$ is

$$
\left(\begin{array}{l}
s \\
p
\end{array}\right)<s^{p}<p^{s}
$$

Thus the number of $G_{x}$ for which there exists a $K_{p}$ so that $K_{p} \cap G_{x}$ has at most $(k+1) s$ edges is less than

$$
C p^{(2 k+3) s} \exp \left(-s^{1+\epsilon-2 \eta}\right)=o(C)
$$

since $\eta<1 / 2 \epsilon$. Thus almost all $G_{x}$ have the following two properties:

(i) There are fewer than $s / t$ closed circuits of $t$ or fewer edges;

(ii) There are at least $(k+1) s$ edges in common with each $K_{p}$. We choose one such $G_{x}$ and delete all edges contained in a circuit of $t$ or fewer edges to give a graph $G$ of girth at least $t$. We have deleted at most $s$ edges from $G_{x}$ so $G$ will have at least $k s>k p$ edges in common with each $K_{p}$.

Lemma 4. Let $H$ be a k-degenerate graph with $p$ vertices. Then $H$ has at most $k p-\left(\begin{array}{c}k+1 \\ 2\end{array}\right)$ edges.

This is Proposition 3 of [7].

Thus any $p$ vertices of $G$ induce a subgraph which is not $k$-degenerate. Therefore $G$ has girth at least $t$ and

$$
\rho(G)>s / p=s^{\eta},
$$

which completes the proof of Theorem 2 .

4. Proof of Theorem 3. Suppose that $\rho(G)>n$. Then there is an induced critical subgraph $H$ of $G$ with $\rho(H)=\rho(G)$ and $\delta(H) \geq(k+1) n$. Clearly $g(H)$ $\geq g(G)$ and $\gamma(H) \leq \gamma(G)$. If $g(H)=\infty$ then $H$ is a finite tree and so $\rho(G)=1$. Otherwise, we embed $H$ in an orientable surface of genus $\gamma(H)$ and from now on $p, q$ and $r$ will denote the number of vertices, edges and faces of $H$. Let 
$g(H)=g$, then every face of $H$ has at least $g$ edges on its boundary, and also $\delta(H) \geq(k+1) n$. Thus

$$
2 q \geq g r \text { and } 2 q \geq(k+1) n p .
$$

Substituting in Euler's formula we have

$$
2-2 \gamma \leq 2-2 \gamma(H)=p-q+r \leq q\left(2 g^{-1}+2(k+1)^{-1} n^{-1}-1\right)
$$

so

$$
q((k+1) g n-2(k+1) n-2 g) \leq 2(k+1) n g(\gamma-1) .
$$

For $\gamma \geq 1$ it follows from (2) that $(k+1) n g-2 n(k+1)-2 g>0$. Hence

$$
p \leq 2 q(k+1)^{-1} n^{-1} \leq 4 g(\gamma-1) /((k+1) n g-2 g-2 n(k+1)) .
$$

Lemma 5. If $t$ is a positive integer such that $g(H) \geq 1+4 t$ and $\delta(H) \geq$ $m \geq 2$ then $p \geq(m-1)^{t} g$.

This is the Corollary to Theorem 1 of [1]. Therefore, writing $b$ for $(k+1) n$,

$$
(h-1)^{t} \leq 4(\gamma-1) /(h g-2 g-2 h),
$$

so that

$$
g \leq 2\left(2(\gamma-1)+h(h-1)^{t}\right) /\left((h-2)(h-1)^{t}\right) .
$$

This completes the proof of Theorem 3 .

\section{REFERENCES}

1. R. J. Cook, Chromatic number and girth, Period. Math. Hungar. (to appear).

2. - Point-arboricity and girth, J. London Math. Soc. (2) 8(1974), 322-324.

3. P. Erdös, Graph theory and probability, Canad. J. Math. 11 (1959), 34-38. MR $21 \# 876$. \#1566.

4. F. Harary, Graph theory, Addison-Wesley, Reading, Mass., 1969. MR 41

5. P. J. Heawood, Map colour theorem, Quart. J. Math. 24 (1890), 332-338.

6. H. V. Kronk, An analogue to the Heawood map-colouring problem, J. London Math. Soc. (2) 1 (1969), 750-752. MR 40 \#4167.

7. D. R. Lick and A. T. White, k-degenerate graphs, Canad. J. Math. 22 (1970), 1082-1096. MR $42 \# 1715$.

8. - The point-partition numbers of closed 2-manifolds, J. London Math. Soc. (2) 4 (1972), 577-583. MR 45 \#5021. 976.

9. P. Turan, On the theory of graphs, Colloq. Math. 3 (1954), 19-30. MR 15,

\section{DEPARTMENT OF MATHEMATICS, UNIVERSITY COLLEGE, CARDIFF, WALES}

Current address: Department of Pure Mathematics, University of Sheffield, Sheffield 10, England 